\title{
Wortschatzerwerb im Unterricht: Wie kann man anders und dabei effektiv mit dem neuen Wortschatz arbeiten?
}

\author{
Vocabulary acquisition: How can we work with the new \\ vocabulary efficiently?
}

\author{
Ivana Zolcerová
}

\begin{abstract}
The subject of this paper is an examination of the ways how vocabulary acquisition can be created. The main aim of this article is to present the phases of the vocabulary acquisition with the efficient activities suitable to each phase. The list of activities is not complete. It should work as an inspiration for other foreign language teachers for their practice.

The article is divided into three parts. In the first part the main terms are defined. The second part is dedicated to the tasks of the teacher by the acquisition of vocabulary. The third part is focused on the phases of vocabulary acquisition and suitable methods for each phase.
\end{abstract}

Key words: vocabulary, phases of vocabulary acquisition, efficient activities

Abstrakt: Thema des vorliegenden Artikels ist die Gestaltung des effektiven Wortschatzerwerbs. Sein Hauptziel besteht darin, den Fremdsprachenlehrern unterschiedliche Lernaktivitäten zu zeigen, die in den verschiedenen Phasen der Wortschatzvermittlung benutzt werden könnten. Der vorliegende Artikel erhebt dabei keineswegs Anspruch auf Vollständigkeit in der Auflistung der Lernaktivitäten. Er soll vielmehr den Fremdsprachenlehrern neue Wege der Wortschatzvermittlung zeigen.

Der Artikel gliedert sich in drei Teile. Nach der Definition der Hauptbegriffe wird auf die Rolle der Lehrkraft in der Wortschatzvermittlung aufmerksam gemacht. Weiter werden die konkreten Phasen der Wortschatzvermittlung geschildert, wobei zu jeder Phase mehrere Aktivitäten der Wortschatzvermittlung erläutert werden.

Schlüsselwörter: Wortschatz, Phasen der Wortschatzvermittlung, effektive Lernaktivitäten

\section{Einleitung}

Seit 1980er Jahren kennt die Fachliteratur den Begriff Wortschatzwende. ${ }^{1}$ In dieser Zeit trat die bis zu diesem Zeitpunkt bevorzugte Grammatikvermittlung, auf der sich die Fremdsprachenlehrwerke sehr oft konzentrierten, in den Hintergrund.

\footnotetext{
${ }^{1}$ Diesen Begriff nutzte F. J. Hausmann in seinem Artikel Die Vokabularisierung des Lehrbuches oder: die Wortschatzwende. Präsentation und Vermittlung von Wortschatz in Lehrwerken für den Französischunterricht. In: Die Neueren Sprachen 86 (1987), S. 426-445. In diesem Aufsatz beschrieb er die benutzten Methoden, wie man die Wortschatzvermittlung in den Lehrwerken unterstützt.
} 
An ihre Stelle kam die Unterstützung der Lernenden beim Wortschatzerwerb in Form von Vokabellisten oder von Übungen zum Wortschatzerwerb. Obwohl seitdem etwa 40 Jahre vergangen sind, bewerten einige Theoretiker ${ }^{2}$ den Stand der Wortschatzvermittlungsforschung immer noch als unzureichend (Teymoortash, 2010). Ein Grund dafür könnte auch die Absenz repräsentativer Studien sein, in denen eine konkrete Lernaktivität der Wortschatzvermittlung mit reliablen Ergebnissen über ihre Effektivität bei der Wortschatzaneignung vorgestellt würde.

Daraus geht hervor, dass die Lehrenden bei der Wortschatzvermittlung größtenteils auf sich selbst gestellt sind und die Lernaktivitäten selbst suchen, probieren und modifizieren müssen. Auf diese Situation versucht der vorliegende Aufsatz zu reagieren. Sein Hauptziel besteht darin, den Fremdsprachenlehrern unterschiedliche Aktivitäten zu zeigen, die in den verschiedenen Phasen der Wortschatzvermittlung benutzt werden könnten. Besonders relevant für die Praxis ist der Bezug der Lernaktivitäten zu der jeweiligen Phase der Wortschatzvermittlung. Viele Lernaktivitäten benutzen Lehrer automatisch, ohne sich bewusst zu werden, welche Phase der Wortschatzvermittlung gerade stattfinden sollte, um den neuen Wortschatz adäquat einzuüben. Der vorliegende Artikel erhebt dabei keineswegs Anspruch auf Vollständigkeit in der Auflistung der Lernaktivitäten. Vielmehr soll er Fremdsprachenlehrer inspirieren und ihnen neue Wege im Umgang mit dem neuen Wortschatz zeigen.

Im Folgenden wird zuerst erklärt, was eigentlich unter dem Begriff Wortschatz $\mathrm{zu}$ verstehen ist und welche Aufgaben der Lehrer bei der Wortschatzvermittlung übernimmt (oder übernehmen sollte). Danach wird der Vorgang der Wortschatzvermittlung dargestellt, wobei zu jeder Phase mehrere Aktivitäten der Wortschatzvermittlung erläutert werden. Die Aktivitäten werden oft mit einem Beispiel dargestellt. Die Beispiele kommen aus dem Bereich Deutsch als Wissenschaftssprache, um zu zeigen, dass solche Aktivitäten den Lernenden auch bei dem Erwerb von abstraktem Wortschatz helfen können.

\section{Ziel der Wortschatzarbeit und die Aufgaben der Lehrenden}

Joanna Targońska (2016, S. 80) versteht unter dem Begriff Wortschatz „ein System von miteinander vernetzten lexikalischen Einheiten." Die lexikalischen Einheiten sind auf verschiedene Weisen verbunden (semantisch, lexikalisch, lexotaktisch). Wenn wir den vermittelten Wortschatz als System wahrnehmen, hilft uns diese Vorstellung, die Phasen der Wortschatzvermittlung auch im Unterricht effektiver zu gestalten (Targońska, 2016).

\footnotetext{
2 Aufgrund der besseren Lesbarkeit wird im Text das generische Maskulinum verwendet. Gemeint sind jedoch immer alle Geschlechter.
} 
Dass „die Wortschatzkenntnisse eine unabdingbare Voraussetzung für einen effizienten und kompetenten Sprachgebrauch und eine erfolgreiche Verständigung darstellen" (Teymoortash, 2010, S. 82), verstehen sowohl Lehrende als auch Lernende. ${ }^{3}$ Ohne den Wortschatz wäre es den Lernenden nicht möglich, in der Fremdsprache zu handeln, was zu den Prinzipien des Fremdsprachenunterrichts (Hippelet al., 2019) gehört.

Multhaup (zit. nach Teymoortash, 2010, S. 82) sieht das Hauptziel der Wortschatzarbeit im „reichen, rezeptiv und/ oder produktiv abrufbaren lexikalischen Wissen in der Fremdsprache." Ein so abstrakt formuliertes Ziel muss jedoch von jedem Lerner und jedem Lehrer spezifiziert werden. Dabei sollen die individuellen Bedürfnisse der Lernenden nicht außer Acht gelassen werden. Erst, wenn ein Lerner eine direkte Verbindung zu seinem eigenen Leben und der eigenen Zukunft sieht, kann er die notwendige Motivation zum Lernen aufbringen. Der Lehrer kann wiederum auf die Lernziele der konkreten Gruppe eingehen und den Wortschatz nach deren Bedürfnissen auswählen.

Dass auch der Lehrer eine zentrale Rolle beim Wortschatzerwerb spielt, erschließt sich aus seinen Tätigkeiten beim Wortschatzerwerb. Die Lehrkraft wählt den Wortschatz aus, präsentiert ihn, vermittelt Bedeutung, bietet die Übungen an, durch die die Lehrkraft bei der Automatisierung, Festigung, Systematisierung und Anwendung des neuen Wortschatzes hilft (De Florio-Hansen, 2006, zit. nach Targońska, 2016). Targońska (2016) macht darauf aufmerksam, dass die Wortschatzarbeit nicht nur eine individuelle Tätigkeit des Lerners ist, sondern von der institutionellen Wortschatzarbeit stark beeinflusst wird. Wie mit dem Wortschatz im Unterricht gearbeitet wird, spiegelt sich auch später in der häuslichen Vorbereitung der Lernenden wider. Die Lehrkraft trägt somit ziemlich viel Verantwortung, ohne dass es ihr oftmals bewusst wird.

\section{Wortschatzvermittlungsphasen und passende Lernaktivitäten}

Nach Doyé (1975, zit. nach Teymoortash, 2010) spielt sich die Wortschatzvermittlung in folgenden Phasen ab:

1. Darbietungsphase - in dieser Phase werden "die Lautgestalt, die Schreibung, die Morphologie, syntaktische Eigenschaften, Valenz und Bedeutung vermittelt" (Teymoortash, 2010, S. 84).

Die Darbietung des neuen Wortschatzes kann durch verschiedene Formen realisiert werden: zweisprachige Vokabellisten, Wortschatz im authentischen Kontext, Lesetexte, Hörtexte, Bilder, Videos u. a.

\footnotetext{
${ }^{3}$ 67\% der Gymnasialstudenten mit Legasthenie sind davon überzeugt, dass der Wortschatzerwerb sehr wichtig ist, obwohl es ihnen Schwierigkeiten bereitet (Rybarczyk, 2014).
} 
In dieser Phase kann die Lehrkraft die Lernenden den neuen Wortschatz mit allen Sinnen erforschen lassen. Je mehr Sinne in diesen Prozess einbezogen werden, desto mehr Synapsen, also Verbindungen im Gehirn, entstehen, und das spätere Erinnern fällt umso leichter (Edelmann \& Wittman, 2019). Ähnlich funktioniert es auch, wenn die Lernenden emotionell angesprochen werden, per Bilder, Geräusche, Überraschung, Videos.

Gerade bei Hör- und Lesetexten, in denen der neue Wortschatz präsentiert wird, sollen die Lernenden den neuen Wortschatz aus dem Kontext erschließen. Nach Clarke und Nation (1980, zit. nach Kostrzewa) kann ein Lerner, der bereits über einen Wortschatz von 3000 Wörtern verfügt, $60-70 \%$ des unbekannten Wortschatzes aus dem Kontext richtig verstehen. Es ist dabei wichtig, zuerst das Wort aus dem Kontext zu erraten, und es erst dann in einem (monolingualen) Wörterbuch nachzuschlagen. Hier hat der Lehrer die Möglichkeit, die Lernenden auf die typischen Wortverbindungen, interessante Wendungen, Phraseme oder Wortbildung aufmerksam zu machen. So lernen die Studierenden Techniken, wie man aus einem neuen Text neben inhaltlichen Informationen auch den neuen Wortschatz gewinnen kann. Diese Methoden sollen im Unterricht bewusst eingeübt werden (Targońska, 2016).

2. Übungsphase: in dieser Phase wird der eingeführte Wortschatz durch Anwendung in verschiedenen Kontexten gefestigt (Doyé 1975, zit. nach Teymoortash, 2010). Auch hier können die Lehrenden unterschiedliche Lernaktivitäten einsetzen, wie weiter mit dem Wortschatz gearbeitet werden kann. Das wichtigste ist, den neuen Wortschatz in das schon existierende System einzugliedern. Hier hilft das mechanische Wörterlernen nicht langfristig. Es ist empfehlenswert, die neuen Vokabeln in das mentale Lexikon nach Begriffsfeldern (r Student, $r$ Mann, $r$ Mensch), Wortfeldern ( $r$ Herzog, $r$ Fürst, $r$ Kaiser, $r$ König - Adelstitel), syntagmatischen Feldern (die Diskussion führen, der Hund bellt - Wörter, die nacheinander vorkommen), Sachfeldern (Einschreibung, Studienabteilung, Universität, Student, Dozent - alles, was zum Thema Studium gehört), Klangfeldern (Brot - Schrott) und Wortfamilien (r Besucher, $r$ Besuch, besuchen) einzugliedern. Auch in dieser Phase existieren verschiedene Möglichkeiten des Übens, z. B. Lückentexte, Ordnen der Vokabeln in Gruppen (nach Synonymen, Antonymen, bzw., Oberbegriffen, Unterbegriffen, Wortfamilien, ...). Dabei erweisen der Lehrkraft gute Dienste auch verschiedene Internetapplikationen, wie etwa Kahoot. Auf der Internetseite kahoot.com kann der Lehrende sehr einfach Quiz herstellen, die die Lernenden als eine Gruppe oder auch individuell spielen können. Jeder Lernende sieht gleich nach der Absendung der Antwort die richtige Lösung und nach dem Wettbewerb sieht er auch seine Platzierung innerhalb der Gruppe.

Durch die digitalen Medien ist die Kontrolle gleich in der Übung eingebaut. Der Lerner kann sich auf diese Weise das Vergessene wieder ins Gedächtnis rufen. Die eingebaute Kontrolle ist auch außerhalb der digitalen Welt mög- 
lich, z. B. durch ein Vokabelpuzzle. Dazu nimmt man ein Bild und teilt es in verschiedene Stücke. An den Rand dieser Teile werden Vokabeln mit ihren Bedeutungen geschrieben. Man schreibt sie am besten so, dass auf einem Teil die Bedeutung eines Wortes steht, auf dem Teil daneben steht dann dieses Wort in der Fremdsprache (auf diese Weise kann man auch Synonyme oder Antonyme verwenden). Wichtig ist, dass die Bedeutungen und das Wort am Rande von zwei Puzzleteilen stehen, die dann zueinander passen. Die Lerner können sich bei der Zuordnung nach den Bedeutungen und den neuen Vokabeln richten oder das Bild zusammenbasteln und dabei die neuen Vokabeln lernen.

Wie schon beim Vokabelpuzzle können durch Anwendung der abwechslungsreichen Aktivitäten verschiedene Lerntypen angesprochen werden. Im Gegensatz zu Vokabellisten erleichtert das Vokabelpuzzle das Lernen vor allem für haptische und visuelle Lerntypen.

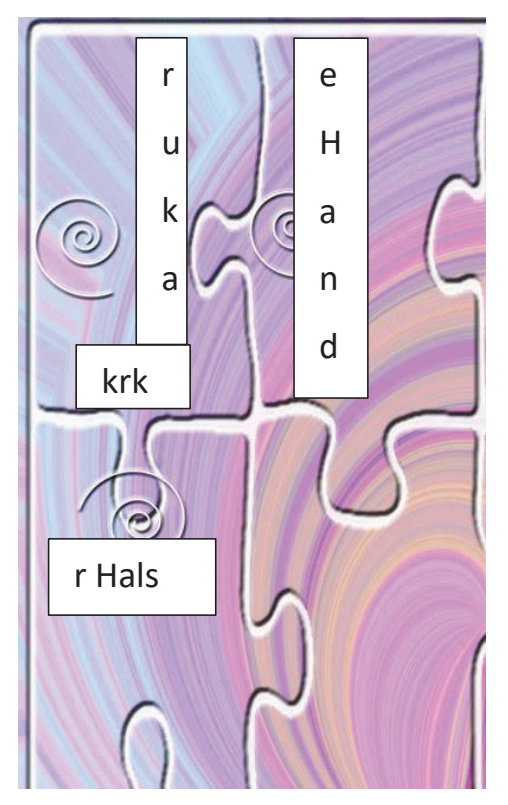

Abb. 1: Vokabelpuzzle

Die nächste Aktivität richtet sich auf solche Lernende, die zu dem Lernen Bewegung brauchen, aber auch auf diejenigen, die gut durch Zuhören lernen können. Die Aktivität Nase, Knie, Bauch ermöglicht uns einen eher als schwierig empfundenen Bestandteil der deutschen Sprache - das Lernen des grammatischen Genus - auf witzige Art und Weise zu üben. Das Prinzip ist sehr einfach: die Lerner wissen, dass es "die" Nase, „der" Bauch und "das" Knie ist. Diese drei Körperteile werden als Code für die Artikel der Substantive benutzt. Immer, wenn der Lehrende ein Substantiv sagt, das den Artikel "die“ hat, fassen sich die Lernenden an der Nase. Bei allen Substantiven, die den Artikel „der“ haben, fassen sich die Studenten wiederum an dem Bauch und bei Substantiven mit "das"-Artikel fassen sie sich an dem Knie. Durch das Zeigen auf diese 
Körperteile lassen die Lernenden den Lehrenden wissen, welchen Artikel sie bei dem genannten Substantiv vermuten. Diese Aktivität braucht zwar nicht viel Platz, aber es ist übersichtlicher, wenn die Lernenden dazu aufstehen.

Eine weitere Aktivität für den haptischen Lerntyp ist eine Variante des Gesellschaftsspiels Activity. Man kann dieses Spiel schon in Anfängergruppen benutzen, wenn man die mündliche Beschreibung zuerst weglässt. Ein Lerner zieht ein Wort, und würfelt, wie er die Bedeutung des Wortes seinem Team vermitteln soll - entweder durch Pantomime, Beschreibung mit anderen Worten oder durch Malen. Diese Aktivität lässt verschiedene Änderungen zu und befeuert auch den Spiel- und Teamgeist der Gruppe.

Für einen überwiegend auditiven Lerntyp ist die Methode Leseschleifegeeignet. Hier bekommt jeder Lerner einen Zettel, auf dem beispielsweise steht:

Lerner A: Ich bin „klug" und suche „sportlich."

Alle Lernenden lesen jetzt ihre Zettel. Auf welchem Zettel steht das Wort „sportlich?“ Der Lernende, der das auf seinem Zettel findet, liest seinen Zettel laut: Ich bin „sportlich“ und suche „ehrlich.“ Wieder lesen alle ihre Zettel durch und meldet sich diejenige Person, bei der auf dem Zettel steht: Ich bin „ehrlich“ und suche usw. Auf diese Weise wird das Vorlesen der Zettel fortgesetzt, bis die Gruppe schließlich zu dem letzten Zettel kommt, auf dem steht: Ich bin „... und suche „klug." So schließt sich der Kreis.

In dieser Aktivität kann man sowohl mit Übersetzungen als auch mit Antonymen, bzw. Synonymen arbeiten und statt Adjektive kann man auch Substantive oder Verben nehmen. In diesem Fall handelt es sich um eine mögliche Aufwärmungsaktivität am Anfang der Unterrichtseinheit.

Beim Vokabelbingo werden Vokabeln durch Schreiben gefestigt. Aus dem zuletzt präsentierten Wortschatz schreibt jeder Lerner neun Vokabeln in eine Tabelle $3 \times 3$.

Eine Beispielstabelle für das Bingo-Spiel:

\section{der Priester}

\begin{tabular}{|l|l|l|}
\hline der Leibeigene & die Zunft & der Adel \\
\hline die Völkerwanderung & die Städtegründung & der Klerus \\
\hline der Kreuzzug & r Mongoleneinfall & r Krieg \\
\hline
\end{tabular}

Das Beispiel zeigt die ausgefüllte Tabelle zum Thema Mittelalter. Das Wort über der Tabelle ist für alle Studierenden in der Gruppe ausgewählt: Dieses Wort sagt der Student laut im Plenum, und die anderen Studenten suchen nach diesem Wort in ihren Tabellen. Wer dieses Wort in der eigenen Tabelle findet, ruft „Bingo“ und bekommt einen Punkt. Gewonnen hat derjenige, der die meisten Punkte gesammelt hat. Man kann in diese Aktivität auch Übersetzungen einbeziehen. In diesem Fall hängt es dann vom Ziel des Lehrers ab, ob man die Vokabeln aus der Fremdsprache in die Muttersprache übersetzt oder umgekehrt. 
Die Zettelaktivität Am Markt setzt die Bewegung der Lehrenden voraus. Der zu wiederholende Wortschatz wird auf kleine Zettel geschrieben. Auf der anderen Seite steht entweder das Äquivalent in der Muttersprache oder die Erklärung in der Fremdsprache. Die Zettel können von der Lehrkraft im Voraus vorbereitet werden oder sie überlässt diese Aufgabe den Lernern. Wenn diese Phase abgeschlossen ist, kann die Gruppe zur zweiten Phase schreiten - dem Spaziergang im Klassenraum. Während des Spaziergangs treffen sich die Lerner in Zweiergruppen. Jeweils zwei Lerner zeigen sich gegenseitig ihre Wörter und jeder erklärt dem anderen das Wort auf dem eigenen Zettel, bzw. wiederholt die Bedeutung des Wortes in der Muttersprache. Die Lernenden tauschen dann ihre Partner. Das führt dazu, dass jeder Lernende die Bedeutung des Wortes aus seinem Zettel mindestens dreimal verschiedenen Partnern erklärt.

Die drauffolgende dritte Phase ist so aufgebaut wie die zweite Phase, nur kommt es hier auch zum Tausch wie auf einem richtigen Markt. Die Studenten zeigen sich ihre Wörter gegenseitig, aber hier soll nun ihr Partner die richtige Bedeutung erraten. Wenn jemand die gefragte Wortbedeutung nicht weiß, hilft der Partner, der auf der anderen Seite des Zettels die richtige Lösung sieht. Nach dieser Kooperation tauschen die Studenten ihre Zettel und gehen weiter mit dem neuen Wort durch den Klassenraum, um einen neuen Partner zu suchen. Wenn jemand aber selbst das Wort auf dem neuen Zettel noch nicht kennt, soll er zuerst die Bedeutung dieses Wortes lernen und darf erst dann einen neuen Partner suchen.

Bei dieser Aktivität fungieren die Lerner selbst als Lehrer, da sie sich gegenseitig neue Wörter erklären. Auf diese Weise ist der Lerner gezwungen, über den zu lernenden Inhalt zu sprechen, was eine andere Aktivierung des Gehirns erfordert, als wenn man sich die Vokabelliste einprägt und wiederholt. Die Interaktion mit anderen Lernern, bzw. mit der Lehrkraft und die damit verbundene Bewegung, führen dazu, dass das einzuprägende Wort mit vielen anderen Eindrücken im Gehirn gespeichert wird. Das auf diese Weise entstandene Netz der verschiedenen Eindrücke, die die Verbindung zu dem neuen Wort aufweisen, ermöglicht dem Lerner ein schnelleres Erinnern, weil zu dem neuen Wort mehr Synapsen führen (Hufeisen et al., 2013).

3. Integrierungsphase. In dieser Phase soll der neue Wortschatz mit den bisherigen Kenntnissen verbunden werden. In dieser Phase sollte auch gesichert werden, dass der neue Wortschatz korrekt verwendet wird (Doyé 1975, zit. nach Teymoortash, 2010).

Die Lehrkraft soll in dieser Phase solche Unterrichtssituationen schaffen, in denen die Lernenden den neuen Wortschatz verwenden können. Dabei sollte die Lehrkraft nicht aus den Augen verlieren, dass alle Lernenden lieber auf schon geübte Wörter zurückgreifen, um auf diese Weise die unangenehme Situation der Unsicherheit oder des Fehlers zu vermeiden. Wenn die Lernenden 
in dieser Phase nicht unterstützt werden und ihnen in dieser Phase nicht ein sicheres Arbeitsklima geschaffen wird, werden sie zur Fossilierung ${ }^{4}$ neigen. Eine sehr oft benutzte und beliebte Aktivität sind Rollenspiele, die auf verschiedenste Weisen variiert werden könnten. Eine beliebte Variante besteht darin, dass sich die Lernenden eine neue Persönlichkeit ausdenken und in dem Rollenspiel nicht als sie selbst sondern als die ausgedachte Person mit einigen ausgeprägten Charakterzügen agieren (z. B. ein sehr zerstreuter oder müder Mensch). Einerseits kann dabei viel Spaß entstehen, andererseits können sich die schüchternen Lerner hinter ihrer Rolle verstecken und müssen nicht persönlich werden.

Ähnlich wie bei Rollenspielen verhält es sich auch bei den moderierten Diskussionen. Man kann sie sehr gut nach landeskundlichen oder historischen Themen in den Unterricht einbinden. Hier zeigen sich uns zwei Möglichkeiten: Zuerst repräsentieren die Lerner ausgewählte Gruppen der Gesellschaft, die entweder bei dem landeskundlichen Thema oder bei dem historischen Ereignis eine wichtige Rolle spielten. Die Studierenden wählen einen Repräsentanten pro Gruppe und denken sich seine persönliche Geschichte aus. Sie überlegen sich, was er dabei gedacht haben könnte und wie er auf die historischen Ereignisse hätte reagieren können.

Beispiel: Das Thema Mauerfall wurde in meinem Seminar mit einer moderierten Diskussion beendet, bei der Studenten in verschiedene Rollen geschlüpft sind. Unter anderem hatten wir ein durch den Mauerbau geteiltes Liebespaar, das sich nach mehr als dreißig Jahren sah, einen Offizier der Staatssicherheit, der in dieser Nacht am 9.11. 1989 in Berlin den Schlagbaum an der Grenze öffnen ließ, einen Rentner, der sich nach dem sozialistischen Regime sehnte, weil er in diesem Regime geachtet wurde, usw.

Die andere Möglichkeit ist, als Diskussionsteilnehmer in die Rolle einer realen Persönlichkeit zu schlüpfen, deren Lebenslauf den Lernenden bekannt sein muss (z. B. Wilson Churchill, Maria Theresia, Greta Thunberg) damit sie deren Entscheidungen und Handlungen auch vor anderen verteidigen können. Diese Form der Diskussion mit wichtigen historischen bzw. gegenwärtigen Persönlichkeiten lässt sich zu jedem aktuellen gesellschaftlichen, aber auch historischen Thema veranstalten.

Weitere Möglichkeiten der Aktivitäten, die bei verschiedenen Themen benutzt werden können, sind z. B. das Verfassen eines eigenen Blogs, die Aufnahme eines Podcasts oder One way Videos (d. h. Aufnahmen, die nur einmal gedreht werden dürfen).

\footnotetext{
${ }^{4}$ Die Fähigkeiten des Lernenden bleiben auf einem niedrigen Niveau und entwickeln sich nicht weiter. Dieses Niveau ermöglicht zwar sehr oft Kommunikation, die Kommunikation ist aber fehlerhaft. Die Fehler entstehen sehr oft durch Interferenzen aus der Muttersprache und werden wiederholt.
} 
Nicht zu unterschätzen ist auch das persönliche Interesse der Lernenden. Die alternativen Aktivitäten, die die Lehrkraft selbst vorschlägt oder mindestens zulässt, ermöglichen den Lernern ihr Lernen selbst zu steuern.

\section{Schlussfolgerung}

Der vorliegende Artikel stellte die Phasen der Wortschatzvermittlung mit passenden Lernaktivitäten dar. Eine gut gewählte Lernaktivität unterstützt den sich gerade abspielenden Vorgang der Wortschatzvermittlungshase und erhöht die Effektivität der Wortschatzvermittlung.

Der Einklang zwischen den Lernaktivitäten und den Vermittlungsphasen ist aber nicht das Einzige, was dabei helfen sollte, dass die Lernenden 70\% des neuen Wortschatzes in der ersten Woche nach seiner Einführung nicht vergessen. Genauso wichtig ist ein Gespräch über Wortschatzaneignungstechniken, die sowohl im Unterricht als auch zu Hause probiert werden können. Ein solches Gespräch unterstützt die Lernenden dabei, Verantwortung für ihr eigenes Lernen zu übernehmen und gibt ihnen effektive Mittel, wie sie ihr eigenes mentales Vokabular aufbauen können.

\section{Literaturverzeichnis}

Clarke, D. F., Nation, I. S. P. (1980). Guessing the meanings of words from context: strategy and techniques. In: System, 8, 211-220. zit. nach KostrzEWA, F. Wortschatzvermittlung im DaF- und DaZ-Unterricht[online]. [vid. 2020-29-1]. Online verfügbar unter: (5) Wortschatzvermittlung im DaF- und DaZ-Unterricht | Frank Kostrzewa - Academia.edu.

De Florio-Hansen, I. (2006). Vom Umgang mit Wörtern. Ergebnisse einer schriftlichen Befragung von Fremdsprachenstudierenden. In: SiepmanN, D. (Hrsg.): Wortschatz und Fremdsprachenlernen. Beiträge zur Fremdsprachenvermittlung, Sonderheft 9, S. 145-191, zit. nach Targońska (2016).

DoYÉ, P. (1971). Systematische Wortschatzvermittlung im Englischunterricht. Schroedel - Lambert Lensing, zit. nach Teymoortash (2010).

Edelmann, W. \& Wittmann, S. (2019). Lernpsychologie: Mit Online-Material. Kösel-Verlag.

Hippel, A., Kulmus, C., Stimm, M. (2019). Didaktik der Erwachsenen und Weiterbildung. utb.

Hufeisen, B., Klippel, J., Pilypaityte, L., Ballweg, S., Drumm, S. (2013). Dll2: Wie lernt man die Fremdsprache Deutsch? Klett-Langenscheidt.

Multhaupt, U. (1995). Psycholinguistik und fremdsprachliches Lernen: von Lehrplänen zu Lernprozessen. Hueber. zit. nach Teymoortash (2010).

RyBARCZYK, R. (2014) Vermittlung von lexikalischen Lernstrategien im DaF-Unterricht in Polen. In: G. FERRARESI, S. LIEBNER (Hg.) SprachBrückenBauen. Universitätsverlag. S. 119-141 [online]. [vid. 2020-29-1]. Online verfügbar unter: (13) (PDF) Vermittlung von lexikalischen Lernstrategien im DaF-Unterricht in Polen unter besonderer Berücksichtigung der Lernenden mit Legasthenie | Renata Rybarczyk - Academia.edu.

TARGoŃSKA, J. (2016). Die Wortschatzarbeit im Fremdsprachenunterricht muss umkehren! Welchen Neuanfang bzw. welche neue Wortschatzwende brauchen wir? In A. D. JARZABEK (Hg.), Anfang. Sprachdidaktische Implikationen für ein neues Lernparadigma (S. 79-93). Könighausen \& Neumann. 
TEYMoortash, N. (2010). Effizienz in der Wortschatzvermittlung. Marburg: Tectum Der Wissenschaftsverlag.

\section{Verzeichnis der Bilder:}

LEEMARLETTo. Puzzle [online]. [vid. 2020-29-1]. Dostupné na internete CC Search (creativecommons.org).

\section{Autorin}

Mgr. Ivana Zolcerová, PhD. e-mail: Ivana.Zolcerova@uniba.sk

Ivana Zolcerová studierte Lehramt Deutsche Sprache und Literatur und Philosophie an der Philosophischen Fakultät der Comenius Universität, Bratislava. 2017 verteidigte sie ihre Dissertation mit dem Titel Inszenierung weiblicher Autorschaft. Die „Volksdichterin“Johanna Ambrosius im Kontext des deutschsprachigen Literaturmarktes Ende des 19. Jahrhunderts am Lehrstuhl für Germanistik, Skandinavistik und Nederlandistik der FiF UK Bratislava. Seitdem arbeitet sie am Lehrstuhl für Sprachen, wo sie Deutsch als Fremdsprache und Fachsprache unterrichtet. In der wissenschaftlichen Arbeit widmet sie sich hauptsächlich der Suche nach effektiven Lehr- und Lernmethoden. Sie erforscht weiter auch die geltenden Beziehungen auf dem Literaturmarkt im 19. Jahrhundert. 